Check for updates

Cite this: RSC Adv., 2019, 9, 30479

Received 4th August 2019

Accepted 17th September 2019

DOI: $10.1039 / c 9 r a 06054 e$

rsc.li/rsc-advances

\section{Simple synthesis of new imidazopyridinone, pyridopyrimidinone, and thiazolopyridinone derivatives and optimization of reaction parameters using response surface methodology $\dagger$}

\begin{abstract}
Fahimeh Sadat Hosseini (D) and Mohammad Bayat (D) *
The reaction between ketene animal/ketene $N, S$-acetals (derived from diamines/cysteamine hydrochloride and 1,1-bis(methylthio)-2-nitroethene) with aromatic aldehydes, and Meldrum's acid led to the title compounds. The reaction conditions were optimized using response surface methodology (RSM). The two independent variables (temperature and water content of aqueous ethanol), and the responses (yield of product and reaction time) were studied. The range of each parameter selected was: $T=25-100{ }^{\circ} \mathrm{C}$ and water $=0-100 \%$. The optimal values were: $T=72{ }^{\circ} \mathrm{C}$ and water $=33 \%$. This work offers significant advantages including use of a green solvent, experimental simplicity, absence of catalyst, a simple workup and purification process, moderate to good yields, and preparation of potentially bioactive compounds.
\end{abstract}

\section{Introduction}

Response surface methodology (RSM) is the most popular optimization method used in recent years. RSM is a powerful mathematical and statistical technique which has been widely used for designing experiments and empirical building models with minimal experimental number. This method is exclusively used to examine the surface, or the relationship between the response and the factors affecting the response. The application of RSM to design optimization is aimed at reducing the cost of expensive analysis methods and their associated numerical noise. ${ }^{1-6}$

Nitrogen and sulfur containing heterocyclic compounds broadly exist in various natural and synthetic products, and play vital roles in medicinal chemistry. ${ }^{7-10}$ Among these heterocycles, pyridine derivatives, such as imidazopyridine, pyridopyrimidine, and thiazolopyridine derivatives have received considerable attention over the past years due to their diverse biological activities and clinical applications. ${ }^{11,12}$ Imidazopyridine derivatives have shown a broad range of interesting biological activities, such as antifungal, antitumor, antiviral, antibacterial, anti-HIV,$^{13}$ antipyretic, analgesic, hypnoselective, anxioselective, anti-inflammatory, anticonvulsant, antiulcer, immunomodulatory ${ }^{14}$ activities; also pyridopyrimidine derivatives ${ }^{15}$ are found to exhibit a broad spectrum of potent antibacterial, antiallergic, antimicrobial, ${ }^{12}$ anti-inflammatory,

Department of Chemistry, Faculty of Science, Imam Khomeini International University, Qazvin, Iran. E-mail: bayat_mo@yahoo.com; m.bayat@sci.ikiu.ac.ir; Tel: +98 28 33780040

$\dagger$ Electronic supplementary information (ESI) available. See DOI: 10.1039/c9ra06054e analgesic, ${ }^{16,17}$ tyrosine kinase, calcium channel antagonists, ${ }^{\mathbf{8}, 18}$ tuberculostatic, antileishmanial, antitumor, ${ }^{19}$ antifolate, antihypertensive, hepatoprotective, ${ }^{20}$ anticancer ${ }^{21}$ properties; and thiazolopyridine derivatives are associated with a wide range of biological activities such as analgesic, antioxidant, ${ }^{11}$ anticancer, ${ }^{22}$ anti-inflammatory, ${ }^{23}$ antihypertensive, ${ }^{24}$ antibacterial, and antifunga ${ }^{25,26}$ properties.

The numerous attractive features of enamines or dienamines derived from the 1,1-bis(methylthio)-2-nitroethene have made them important intermediates for the construction of a wide variety of $\mathrm{S}$ - and N-heterocyclic systems. ${ }^{27-33}$ Hence during recent years, they have been used for the synthesis of various fused heterocycles and drug-like compounds. For examples in 2013 Bazgir et al. described a one-pot, four-component synthesis of a novel class of functionalized imidazo[1,2-a]pyridine derivatives starting from readily available inputs including diverse diamines, 1,1-bis(methylthio)-2-nitroethene, aldehydes and activated methylene compounds in EtOH under reflux conditions (Scheme 1, Entry a). ${ }^{34}$ In 2014 Yildirim et al. reported a base-catalyzed one-pot cyclocondensation reaction of acryloyl and cinnamoyl chlorides with $\beta$-nitroenamine derivatives under mild basic conditions, which affords target thiazolopyridinone or imidazopyridinone derivatives (Scheme 1, Entry b). ${ }^{35}$ In 2017 and 2018 we developed an efficient, one-pot, multi-component synthesis of imidazopyridine, pyridopyrimidine, thiazolopyridine, and oxazolopyridine fused heterocyclic systems via reaction of various diamines, cysteamine hydrochloride, ethanolamine, 1,1-bis(methylthio)-2-nitroethene, cyanoacetohydrazide and aromatic aldehydes in ethanol at reflux (Scheme 1, Entry c). ${ }^{36,37}$ Herein we report synthesis of a new class of imidazopyridinone, pyridopyrimidinone, and 
a) Bazgir's work

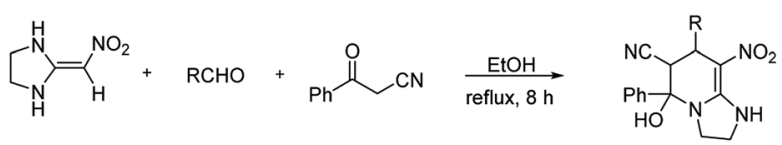

b) Yildirim's work

$$
\text { bese, solvent }
$$

c) Our previous work

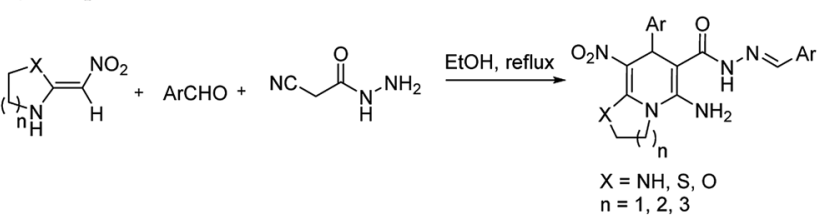

d) This work

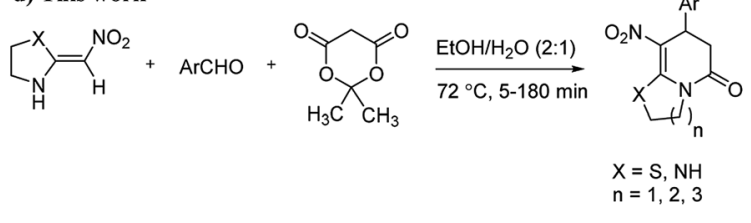

Scheme 1 Use of enamines derived from 1,1-bis(methylthio)-2nitroethene in organic synthesis.

thiazolopyridinone derivatives through a simple one-pot, multicomponent reaction of diverse diamines or cysteamine hydrochloride, 1,1-bis(methylthio)-2-nitroethene, aromatic aldehydes, and 2,2-dimethyl-1,3-dioxane-4,6-dione (Meldrum's acid) in ethanol/water $(2: 1)$ at $72{ }^{\circ} \mathrm{C}$ (Scheme 1, Entry d). RSM in conjunction with a central composite design was used for modeling and optimizing the synthetic process.

\section{Result and discussion}

The one-pot, multi-component reaction of various diamines or cysteamine hydrochloride 1 with 1,1-bis(methylthio)-2nitroethene 2, aromatic aldehydes 3, and 2,2-dimethyl-1,3dioxane-4,6-dione (Meldrum's acid) 4, in EtOH/ $\mathrm{H}_{2} \mathrm{O}(2: 1)$ at $72{ }^{\circ} \mathrm{C}$, gave imidazopyridinone, pyridopyrimidinone, and thiazolopyridinone derivatives $\mathbf{5}$ in moderate to good yields (Scheme 2).
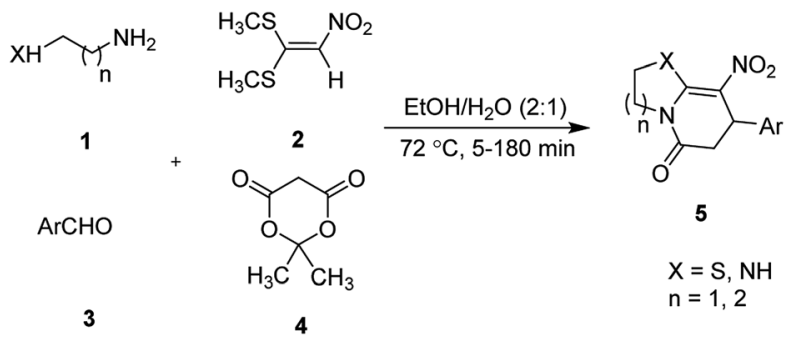

Scheme 2 Synthetic scheme for the products $5 a-0$.
A reaction condition is optimized using response surface methodology (RSM). A central composite design with five replicates at the center point requiring 13 treatments was used. The two independent variables (temperature $(A)$ and water content of aqueous ethanol $(B)$ ), levels (Table 1 ), and the results obtained after running the experiments are represented in Table 2 . The following responses were analyzed: yield of product $\left(R_{1}\right)$ and reaction time $\left(R_{2}\right)$.

Optimum conditions with respect to yield, purity of product and reaction time were as follow: temperature $72{ }^{\circ} \mathrm{C}$, water content of aqueous ethanol $33 \%$. Verification experiments, carried out at the predicted conditions showed values reasonably close to those predicted and further confirmed the adequacy of predicted models.

On the basis of the central composite design, the cubic model relationship between the experimental yield $\left(R_{1}\right)$ and the process variables (temperature $(A)$ and water content of aqueous ethanol $(B))$ in coded units is obtained from eqn (1).

$$
\begin{aligned}
R_{1}= & 82.60+11.31 A-18.74 B-0.25 A B-10.55 A^{2} \\
& -9.30 B^{2}+11.49 A^{2} B-5.06 A B^{2}
\end{aligned}
$$

where $R_{1}$ represents the experimental yield of the reaction, then $A$ (temperature) and $B$ (water content of aqueous ethanol) are the coded variables in the reaction.

Fig. 1 illustrates the good linear correlation between the actual and predicted yield and sufficient accuracy of the forecast values. Therefore, the models can be used to predict the yield of product successfully.

The ANOVA for response surface cubic models are reported in Table 3. The model $F$-value of 189.50 implies the model is significant. The "Lack of Fit $F$-value" is 0.71 then it is not significant relative to the pure error. Non-significant lack of fit is good and we want the model to fit. The "Pred $R$-Squared" of 0.9586 is in reasonable agreement with the "Adj $R$-Squared" of 0.9910 ; i.e., the deviation is about 0.3 .

The effects of temperature and water content of aqueous ethanol on the total reaction yield are shown in Fig. 2. The total yield varied from $37 \%$ to $90 \%$. As temperature increased, the yield increased. The changes in yield versus the water content of aqueous ethanol is minor compared to that of temperature. The highest yield (90\%) was obtained at $62.5{ }^{\circ} \mathrm{C}$ and at $0 \%$ water. The lowest yield (37\%) was at $62.5^{\circ} \mathrm{C}$ and at $100 \%$ water.

The effects of temperature and the water content of aqueous ethanol on the total reaction time are shown in Fig. 3. The analysis of total reaction time $\left(R_{2}\right)$ showed the significant Lack of fit and it is not good. So the temperature and water content of aqueous ethanol have no effect on the reaction time and the total reaction time was almost identical and short in any case.

Table 1 Selected variables and levels used in central composite design

\begin{tabular}{lllllll}
\hline & & \multicolumn{4}{c}{ Levels } \\
\cline { 5 - 7 } Variables & Code & Units & -1 & 0 & +1 \\
\hline Reaction temperature & $A$ & ${ }^{\circ} \mathrm{C}$ & 25 & 62.5 & 100 \\
Water content of aqueous ethanol & $B$ & $\%$ & 0 & 50 & 100
\end{tabular}


Table 2 Process variables and experimental data for two factors, three levels response surface design ${ }^{a}$

\begin{tabular}{lrrlr}
\hline Run & $A\left({ }^{\circ} \mathrm{C}\right)$ & $B(\%)$ & $R_{1}(\%)$ & $R_{2}(\mathrm{~min})$ \\
\hline 1 & 62.50 & 50.00 & 80 & 7 \\
2 & 35.98 & 85.36 & 50 & 5 \\
3 & 89.02 & 14.64 & 77 & 5 \\
4 & 62.50 & 50.00 & 83 & 6 \\
5 & 25.00 & 50.00 & 45 & 11 \\
6 & 62.50 & 50.00 & 84 & 5 \\
7 & 100.00 & 50.00 & 77 & 5 \\
8 & 62.50 & 0.00 & 90 & 5 \\
9 & 62.50 & 50.00 & 84 & 5 \\
10 & 89.02 & 85.36 & 62 & 6 \\
11 & 62.50 & 100.00 & 37 & 11 \\
12 & 62.50 & 50.00 & 82 & 5 \\
13 & 35.98 & 14.64 & 64 & 5
\end{tabular}

${ }^{a} A=$ temperature $\left({ }^{\circ} \mathrm{C}\right), B=$ water content of aqueous ethanol (\%), $R_{1}=$ yield of reaction, $R_{2}=$ reaction time.

The scope of the reaction substrate was explored under optimized conditions. Several kind of aromatic aldehydes, and various diamines were tolerated. The reaction was completed after 5-180 min to afford corresponding S- and N-heterocyclic systems 5a-p, in moderate to good yields (50-89\%). As shown in Table 4, reactions with diamines, usually resulted in good yields and short reaction times. Reactions with cysteamine hydrochloride, resulted in low yields and long reaction times. Also the structures of the aldehydes had obvious influence on the yield of product and reaction time. Reactions involving aromatic aldehydes usually went smoothly and resulted in good yields and short reaction times. Reaction involving heteroaromatic aldehyde (such as furfural) and aliphatic aldehyde (such as butyraldehyde), on the other hand, were normally slow and resulted in low and trace yields, respectively. When the 4- pyridinecarboxaldehyde was used the reaction did not work. The substitutions of the aromatic aldehydes had some influence on the yields and reaction times. Reaction with aromatic aldehydes carrying an electron-withdrawing group, such as chloro and nitro groups, usually resulted in good yields and short reaction times; those carrying electron-donating groups such as methoxy group, resulted in relatively poorer yields. The reaction did not work, when the reaction was performed using ethanolamine, cyclohexane-1,2-diamine and benzene-1,2diamine.

The structures of products were fully characterized using their infrared (IR), mass spectrometry, proton hydrogen-1 nuclear magnetic resonance ( ${ }^{1} \mathrm{H}$ NMR), carbon-13 nuclear magnetic resonance $\left({ }^{13} \mathrm{C}\right.$ NMR $)$ spectra. For example, the mass spectrum of 5 a displayed the molecular ion peak at $\mathrm{m} / \mathrm{z} 293$ with frequency of $53 \%$ which was in accordance with the proposed structure. The IR spectrum of this structure indicated absorption bands due to the $\mathrm{NH}$ stretching $\left(3231 \mathrm{~cm}^{-1}\right)$ as well as bands at 2922, 1704, 1623, 1486 and $1337 \mathrm{~cm}^{-1}$ due to the $\mathrm{CH}$, $\mathrm{C}=\mathrm{O}, \mathrm{C}=\mathrm{C}$ and $\mathrm{NO}_{2}$ groups. The ${ }^{1} \mathrm{H}$ NMR spectrum of $\mathbf{5 a}$ showed doublet of doublet for the $\mathrm{CH}_{2}$ group $(\delta 2.55,3.21 \mathrm{ppm}$, ${ }^{2} J_{\mathrm{HH}}=15.6 \mathrm{~Hz},{ }^{3} \mathrm{~J}_{\mathrm{HH}}=7.8 \mathrm{~Hz}$ ), multiplets for $\mathrm{CH}_{2} \mathrm{NH}$ and $\mathrm{CH}_{2} \mathrm{~N}$ groups $(\delta 3.69-3.97 \mathrm{ppm})$, doublet for $\mathrm{CH}$ group $(\delta 4.52 \mathrm{ppm}$, $\left.{ }^{3} J_{\mathrm{HH}}=7.5 \mathrm{~Hz}\right)$, two doublets for the aromatic region $(\delta 7.16$, $\left.7.32 \mathrm{ppm},{ }^{3} \mathrm{JHH}_{\mathrm{HH}}=8.4 \mathrm{~Hz}\right)$ and one singlet for $\mathrm{NH}$ group $(\delta 9.74$ $\mathrm{ppm})$. The ${ }^{1} \mathrm{H}$-decoupled ${ }^{13} \mathrm{C}$ NMR spectrum of 5a showed 11 distinct resonances. There are three signals for $\mathrm{CH}$ and $\mathrm{CH}_{2}$ groups ( $\delta 37.0,43.0,43.9 \mathrm{ppm})$, and signals at 106.3 and $167.6 \mathrm{ppm}$, which were assigned $\mathrm{C}-\mathrm{NO}_{2}$ and $\mathrm{C}=\mathrm{O}$ groups, respectively. The ${ }^{1} \mathrm{H}$ and ${ }^{13} \mathrm{C}$ NMR spectra of $5 \mathbf{b}-\mathbf{p}$ are similar to those of 5a except for the aryl and diamine moieties, which exhibited characteristic signals with appropriate chemical shifts (see the ESI $\dagger$ ).

An acceptable reaction mechanism for this one-pot, multicomponent reaction is designated in Scheme 3. The one-pot

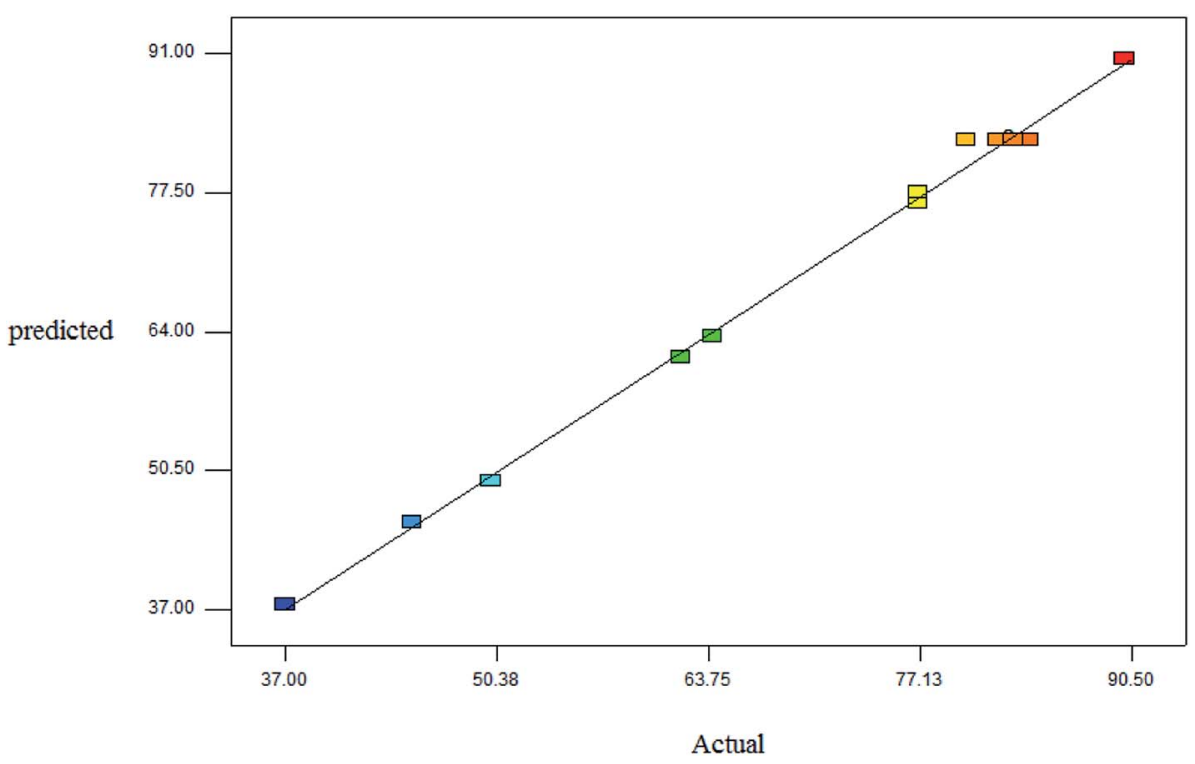

Fig. 1 Linear correlation between the actual and predicted yield. 
Table 3 ANOVA for response surface cubic model

\begin{tabular}{|c|c|c|c|c|c|}
\hline Source & Sum of square & Degree of freedom & Mean square & $F$ value & $p$-Value, prob $>F$ \\
\hline Model & 3501.88 & 7 & 500.27 & 189.50 & $<0.0001$ significant \\
\hline$A$-temperature & 512.00 & 1 & 512.00 & 193.94 & $<0.0001$ \\
\hline$A B$ & 0.25 & 1 & 0.25 & 0.095 & 0.7707 \\
\hline$A^{2}$ & 774.28 & 1 & 772.28 & 293.29 & $<0.0001$ \\
\hline$B^{2}$ & 601.67 & 1 & 601.67 & 227.91 & $<0.0001$ \\
\hline$A^{3}$ & 0.000 & 0 & & & \\
\hline$B^{3}$ & 0.000 & 0 & & & \\
\hline Residual & 13.20 & 5 & 2.64 & & \\
\hline Lack of fit & 2.0 & 1 & 2.00 & 0.71 & \\
\hline Pure error & 11.20 & 4 & 2.80 & & 0.4456 not significant \\
\hline Correlation total & 3515.08 & 12 & & & \\
\hline
\end{tabular}

protocol started with the reaction of various diamines or cysteamine hydrochloride 1 and 1,1-bis(methylthio)-2nitroethene 2 to afford enamine 6 . Also condensation reaction between 2,2-dimethyl-1,3-dioxane-4,6-dione (Meldrum's acid) and the aromatic aldehydes gave adduct 7 , which then reacted with enamine 6 to form intermediate 8. Compound 10 was formed from $\mathbf{8}$ through imine-enamine tautomerism and cyclocondensation. Finally, decarboxylation of $\mathbf{1 0}$ yielded the product 5 .

\section{Experimental}

\section{General}

The various diamines, cysteamine hydrochloride, 1,1bis(methylthio)-2-nitroethene, aldehydes, 2,2-dimethyl-1,3dioxane-4,6-dione (Meldrum's acid) and solvents were purchased from Sigma-Aldrich chemical company and were used as received without further purification. Melting points were determined with an electrothermal 9100 apparatus. Infrared (IR) spectra were recorded on a Bruker Tensor 27 spectrometer. Nuclear magnetic resonance (NMR) spectra were obtained on a Bruker DRX-300 Avance instrument (300 MHz for ${ }^{1} \mathrm{H}$ and 75.4 MHz for ${ }^{13} \mathrm{C}$ ) with DMSO as solvent. Chemical shifts are expressed in parts per million ( $\mathrm{ppm}$ ), and coupling constant $(J)$ are reported in hertz $(\mathrm{Hz})$. Elemental analyses for $\mathrm{C}, \mathrm{H}$ and $\mathrm{N}$ were performed using a PerkinElmer 2004 series [II] CHN elemental analyzer. Mass spectra were recorded with an Agilent 5975C VL MSD with Triple-Axis Detector operating at an ionization potential of $70 \mathrm{eV}$.

\section{General procedure for the synthesis of product 5}

Synthesis of imidazopyridinone and pyridopyrimidinone derivatives. The mixtures of various diamines (1 mmol), 1,1bis(methylthio)-2-nitroethene $(0.165 \mathrm{~g}, 1 \mathrm{mmol})$ and $10 \mathrm{~mL}$ $\mathrm{EtOH} / \mathrm{H}_{2} \mathrm{O}(2: 1)$ at $72{ }^{\circ} \mathrm{C}$ in a $50 \mathrm{~mL}$ flask was stirred for $6 \mathrm{~h}$. After completion of the reaction (monitored by thin-layer chromatography, hexane/ethyl acetate $1: 1$ ), aromatic aldehyde (1 mmol), 2,2-dimethyl-1,3-dioxane-4,6-dione (Meldrum's acid) $(0.144 \mathrm{~g}, 1 \mathrm{mmol})$ were added to the reaction mixture, and it was stirred under optimized condition for the time given in Table 4 . Then, the reaction mixture was cooled to room temperature and filtered to give the crude product. The solid was washed with ethanol to give product in good yields.

Synthesis of thiazolopyridinone derivatives. The mixtures of cysteamine hydrochloride $(0.113 \mathrm{~g}, 1 \mathrm{mmol}), 1$,1-bis(methylthio)-2-
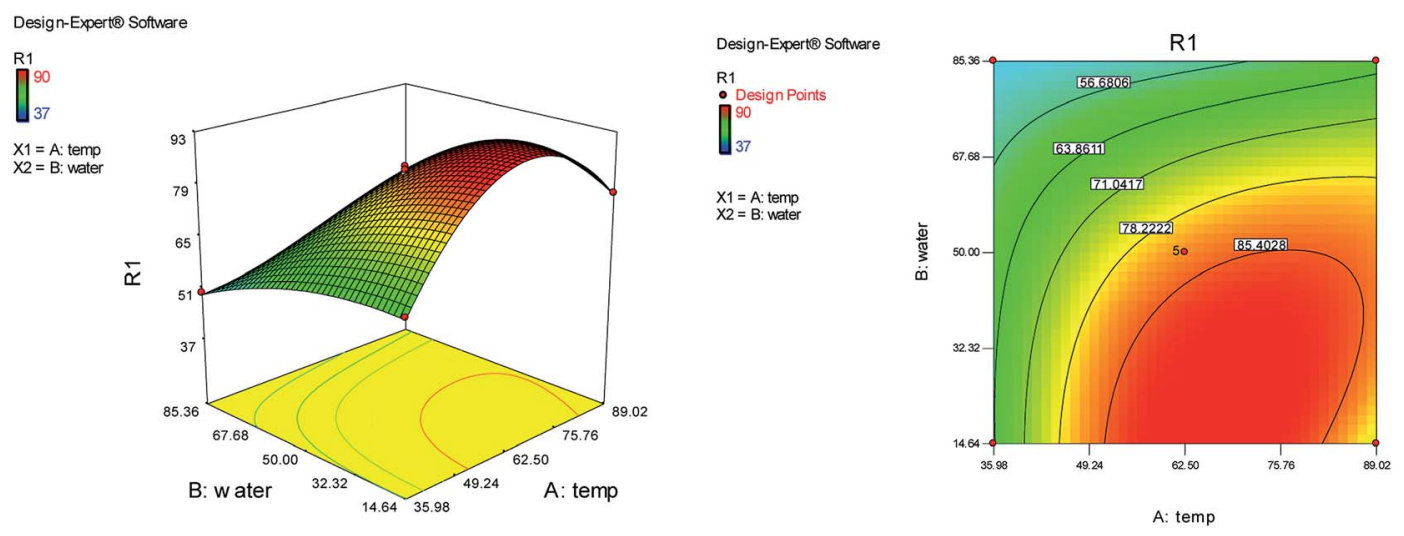

Fig. 2 The effects of temperature and water content of aqueous ethanol on the total reaction yield. 
View Article Online

RSC Advances

Paper

Table 4 (Contd.)

Entry Aldehyde $^{a}$<smiles>NCCS</smiles>

9<smiles>O=Cc1ccc([N+](=O)[O-])cc1</smiles><smiles>NCCS</smiles>

120

60

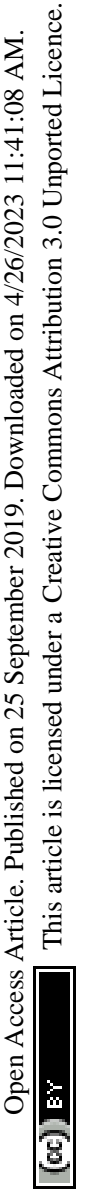

10<smiles>O=Cc1ccccc1</smiles><smiles>NCCN</smiles>

11<smiles>COc1ccc(C=O)cc1</smiles>

12<smiles>COc1cccc(C=O)c1</smiles><smiles>NCCN</smiles>

13<smiles>O=Cc1ccco1</smiles><smiles>NCCN</smiles><smiles>O=C1CC(c2ccc([N+](=O)[O-])cc2)C([N+](=O)[O-])=C2SCCN12</smiles>

150

57

$5 i$<smiles>O=C1CC(c2ccccc2)C([N+](=O)[O-])=C2NCCN12</smiles>

$5 j$<smiles>COc1ccc(C2CC(=O)N3CCNC3=C2[N+](=O)[O-])cc1</smiles>

25

76

$\mathrm{H}_{2} \mathrm{~N} \Upsilon \mathrm{NH}_{2}$ 15

70

$5 k$<smiles>COc1cccc(C2CC(=O)N3CCNC3=C2[N+](=O)[O-])c1</smiles>

25

74

SI<smiles>O=C1CC(c2ccco2)C([N+](=O)[O-])=C2NCCN12</smiles>

150

57

14<smiles>COc1cccc(C=O)c1</smiles>

180

50

$5 n$

30484 | RSC Adv., 2019, 9, 30479-30488

This journal is (c) The Royal Society of Chemistry 2019 
Table 4 (Contd.)

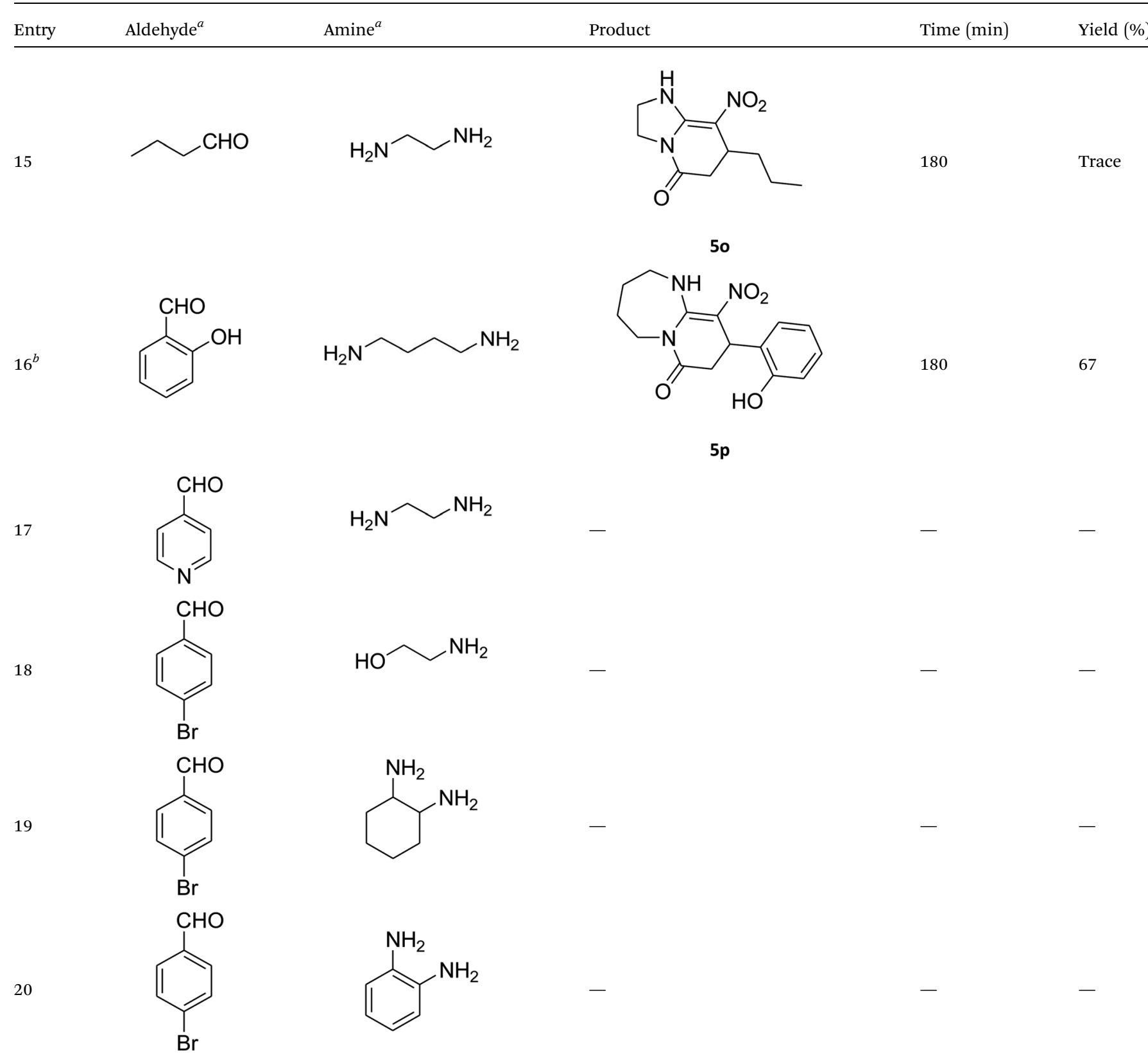

${ }^{a}$ Amine (1 mmol), 1,1-bis(methylthio)-2-nitroethene (1 mmol), aromatic aldehyde $(1 \mathrm{mmol})$ and 2,2-dimethyl-1,3-dioxane-4,6-dione (Meldrum's acid) $(1 \mathrm{mmol})$ were used. ${ }^{b}$ Ref. 38.

nitroethene (0.165 g, $1 \mathrm{mmol}), 10 \mathrm{~mL} \mathrm{EtOH} / \mathrm{H}_{2} \mathrm{O}(2: 1)$, and $\mathrm{Et}_{3} \mathrm{~N}$ $(140 \mu \mathrm{L}, 1 \mathrm{mmol})$ at $72{ }^{\circ} \mathrm{C}$ in a $50 \mathrm{~mL}$ flask was stirred for $5 \mathrm{~h}$. After completion of the reaction (monitored by thin-layer chromatography, hexane/ethyl acetate $1: 1$ ), aromatic aldehyde (1 mmol), 2,2dimethyl-1,3-dioxane-4,6-dione (Meldrum's acid) (0.144 g, $1 \mathrm{mmol}$ ) were added to the reaction mixture, and it was stirred under optimized condition for the time given in Table 4 . The reaction mixture was monitored by thin layer chromatography (hexane/ethyl acetate $1: 1$ ); after completion of the reaction, the reaction mixture was cooled to room temperature and the product (as colorless crystals) was filtered. The solid was washed with ethanol to give product in moderate yields.

7-(4-Chlorophenyl)-2,3,6,7-tetrahydro-8-nitroimidazo[1,2-a]pyridin-5(1H)-one (5a). White solid; yield: $0.257 \mathrm{~g}(88 \%)$; mp 299$301{ }^{\circ} \mathrm{C} .{ }^{1} \mathrm{H}$ NMR (300 MHz, DMSO- $\left.d_{6}\right): \delta 2.55\left(\mathrm{~d},{ }^{2} J_{\mathrm{HH}}=15.6 \mathrm{~Hz}\right.$, $\left.1 \mathrm{H}, \mathrm{CH}_{2}\right), 3.21\left(\mathrm{dd},{ }^{2} J_{\mathrm{HH}}=15.6 \mathrm{~Hz},{ }^{3} J_{\mathrm{HH}}=7.8 \mathrm{~Hz}, 1 \mathrm{H}, \mathrm{CH}_{2}\right.$ ), 3.69-3.97 (m, $4 \mathrm{H}, \mathrm{CH}_{2} \mathrm{NH}$ and $\left.\mathrm{CH}_{2} \mathrm{~N}\right), 4.52\left(\mathrm{~d},{ }^{3} J_{\mathrm{HH}}=7.5 \mathrm{~Hz}, 1 \mathrm{H}\right.$, $\mathrm{CH}), 7.16\left(\mathrm{~d},{ }^{3} J_{\mathrm{HH}}=8.4 \mathrm{~Hz}, 2 \mathrm{H}, \mathrm{Ar}\right), 7.32\left(\mathrm{~d},{ }^{3} J_{\mathrm{HH}}=8.4 \mathrm{~Hz}, 2 \mathrm{H}\right.$, $\mathrm{Ar}), 9.74(\mathrm{~s}, 1 \mathrm{H}, \mathrm{NH}) .{ }^{13} \mathrm{C} \mathrm{NMR}\left(75.4 \mathrm{MHz}, \mathrm{DMSO}-d_{6}\right): \delta 37.0$, $43.0,43.9,106.3,128.8,129.0,131.9,141.9$, 153.0, 167.6. IR 
$(\mathrm{KBr})\left(\nu_{\max } / \mathrm{cm}^{-1}\right): 3231(\mathrm{NH}), 2922(\mathrm{CH}), 1704(\mathrm{C}=\mathrm{O}), 1623(\mathrm{C}=$ C), 1486 and $1337\left(\mathrm{NO}_{2}\right), 1214(\mathrm{C}-\mathrm{N})$. MS (EI, $\left.70 \mathrm{eV}\right): m / z(\%)=$ $293\left(\mathrm{M}^{+}, 53\right), 276$ (42), 240 (100), 218 (23), 182 (59), 151 (35), 136 (45), 70 (18), 42 (14). Anal. calc. for $\mathrm{C}_{13} \mathrm{H}_{12} \mathrm{ClN}_{3} \mathrm{O}_{3}$ (293.71): C, 53.16; H, 4.12; N, 14.31. Found: C, 53.01; H, 4.51; N, 14.03.

7-(4-Bromophenyl)-2,3,6, 7-tetrahydro-8-nitroimidazo[1,2-a]pyridin-5(1H)-one (5b). White solid; yield: $0.294 \mathrm{~g}$ (87\%); mp 305$307{ }^{\circ} \mathrm{C} .{ }^{1} \mathrm{H}$ NMR $\left(300 \mathrm{MHz}, \mathrm{DMSO}-d_{6}\right): \delta 2.55\left(\mathrm{~d},{ }^{2} J_{\mathrm{HH}}=16.6 \mathrm{~Hz}\right.$, $\left.1 \mathrm{H}, \mathrm{CH}_{2}\right), 3.21\left(\mathrm{dd},{ }^{2} J_{\mathrm{HH}}=16.6 \mathrm{~Hz},{ }^{3} J_{\mathrm{HH}}=7.8 \mathrm{~Hz}, 1 \mathrm{H}, \mathrm{CH}_{2}\right.$ ), 3.69-3.97 (m, $4 \mathrm{H}, \mathrm{CH}_{2} \mathrm{NH}$ and $\left.\mathrm{CH}_{2} \mathrm{~N}\right), 4.51\left(\mathrm{~d},{ }^{3} J_{\mathrm{HH}}=7.8 \mathrm{~Hz}, 1 \mathrm{H}\right.$, $\mathrm{CH}), 7.10\left(\mathrm{~d},{ }^{3} J_{\mathrm{HH}}=7.2 \mathrm{~Hz}, 2 \mathrm{H}, \mathrm{Ar}\right), 7.45\left(\mathrm{~d},{ }^{3} J_{\mathrm{HH}}=7.2 \mathrm{~Hz}, 2 \mathrm{H}\right.$, $\mathrm{Ar}), 9.73(\mathrm{~s}, 1 \mathrm{H}, \mathrm{NH}) .{ }^{13} \mathrm{C}$ NMR $\left(75.4 \mathrm{MHz}, \mathrm{DMSO}-d_{6}\right): \delta 37.1$, 43.1, 44.0, 106.3, 120.3, 129.1, 131.9, 142.3, 153.0, 167.6. IR $(\mathrm{KBr})\left(\nu_{\max } / \mathrm{cm}^{-1}\right): 3224(\mathrm{NH}), 2921(\mathrm{CH}), 1699(\mathrm{C}=\mathrm{O}), 1623(\mathrm{C}=$ C), 1486 and $1337\left(\mathrm{NO}_{2}\right), 1215(\mathrm{C}-\mathrm{N})$. MS (EI, $\left.70 \mathrm{eV}\right): m / z(\%)=$ 337 ( $\left.\mathrm{M}^{+}-1,25\right), 320$ (22), 291 (58), 240 (100), 212 (12), 182 (51), 136 (29), 116 (33), 70 (49), 42 (22). Anal. calc. for $\mathrm{C}_{13} \mathrm{H}_{12} \mathrm{BrN}_{3} \mathrm{O}_{3}$ (338.16): C, 46.17; H, 3.58; N, 12.43. Found: C, 46.52; H, 3.21; N, 12.12 .

2,3,6,7-Tetrahydro-8-nitro-7-(4-nitrophenyl)imidazo[1,2-a]pyridin-5(1H)-one (5c). Yellow solid; yield: $0.270 \mathrm{~g}$ (89\%); mp 264$266{ }^{\circ} \mathrm{C} .{ }^{1} \mathrm{H}$ NMR $\left(300 \mathrm{MHz}, \mathrm{DMSO}-d_{6}\right): \delta 2.59\left(\mathrm{~d},{ }^{2} J_{\mathrm{HH}}=15.6 \mathrm{~Hz}\right.$, $\left.1 \mathrm{H}, \mathrm{CH}_{2}\right), 3.26\left(\mathrm{dd},{ }^{2} J_{\mathrm{HH}}=15.6 \mathrm{~Hz},{ }^{3} J_{\mathrm{HH}}=8.1 \mathrm{~Hz}, 1 \mathrm{H}, \mathrm{CH}_{2}\right)$, 3.71-3.99 (m, $4 \mathrm{H}, \mathrm{CH}_{2} \mathrm{NH}$ and $\left.\mathrm{CH}_{2} \mathrm{~N}\right), 4.68\left(\mathrm{~d},{ }^{3} J_{\mathrm{HH}}=7.5 \mathrm{~Hz}, 1 \mathrm{H}\right.$, $\mathrm{CH}), 7.44\left(\mathrm{~d},{ }^{3} J_{\mathrm{HH}}=8.7 \mathrm{~Hz}, 2 \mathrm{H}, \mathrm{Ar}\right), 8.12\left(\mathrm{~d},{ }^{3} J_{\mathrm{HH}}=8.7 \mathrm{~Hz}, 2 \mathrm{H}\right.$, $\mathrm{Ar}), 9.79(\mathrm{~s}, 1 \mathrm{H}, \mathrm{NH}) .{ }^{13} \mathrm{C}$ NMR $\left(75.4 \mathrm{MHz}, \mathrm{DMSO}-d_{6}\right): \delta 37.6$, 43.1, 44.0, 105.8, 124.3, 128.3, 146.9, 150.8, 153.0, 167.2. IR $(\mathrm{KBr})\left(\nu_{\max } / \mathrm{cm}^{-1}\right): 3227(\mathrm{NH}), 2923(\mathrm{CH}), 1712(\mathrm{C}=\mathrm{O}), 1628(\mathrm{C}=$
C), 1520 and $1345\left(\mathrm{NO}_{2}\right), 1219(\mathrm{C}-\mathrm{N})$. Anal. calc. for $\mathrm{C}_{13} \mathrm{H}_{12} \mathrm{~N}_{4} \mathrm{O}_{5}$ (304.26): C, 51.32; H, 3.98; N, 18.41. Found: C, 51.56; H, 3.64; N, 18.16.

7-(3-Fluorophenyl)-2,3,6,7-tetrahydro-8-nitroimidazo[1,2-a]pyridin-5(1H)-one (5d). White solid; yield: $0.207 \mathrm{~g}$ (75\%); mp 253$255{ }^{\circ} \mathrm{C} .{ }^{1} \mathrm{H}$ NMR $\left(300 \mathrm{MHz}, \mathrm{DMSO}-d_{6}\right): \delta 2.59\left(\mathrm{~d},{ }^{2} J_{\mathrm{HH}}=16.6 \mathrm{~Hz}\right.$, $\left.1 \mathrm{H}, \mathrm{CH}_{2}\right), 3.20\left(\mathrm{~m},{ }^{2} J_{\mathrm{HH}}=16.6 \mathrm{~Hz},{ }^{3} J_{\mathrm{HH}}=8.1 \mathrm{~Hz}, 1 \mathrm{H}, \mathrm{CH}_{2}\right)$, 3.70-3.94 (m, $4 \mathrm{H}, \mathrm{CH}_{2} \mathrm{NH}$ and $\left.\mathrm{CH}_{2} \mathrm{~N}\right), 4.55\left(\mathrm{~d},{ }^{3} J_{\mathrm{HH}}=7.5 \mathrm{~Hz}, 1 \mathrm{H}\right.$, $\mathrm{CH}$ ), 6.96-7.06 (m, 3H, Ar), 7.28-7.35 (m, 1H, Ar), 9.75 (s, 1H, $\mathrm{NH}) .{ }^{13} \mathrm{C}$ NMR $\left(75.4 \mathrm{MHz}, \mathrm{DMSO}-d_{6}\right): \delta 37.4,43.1,44.0,106.2$, $113.8\left(\mathrm{~d},{ }^{2} J_{\mathrm{CF}}=21 \mathrm{~Hz}\right), 114.2\left(\mathrm{~d},{ }^{2} J_{\mathrm{CF}}=21 \mathrm{~Hz}\right), 122.8,131.1$, 145.9, 153.1, $162.8\left(\mathrm{~d},{ }^{1} J_{\mathrm{CF}}=244 \mathrm{~Hz}\right), 167.6$. Anal. calc. for $\mathrm{C}_{13} \mathrm{H}_{12} \mathrm{FN}_{3} \mathrm{O}_{3}$ (277.25): C, 56.32; H, 4.36; N, 15.16. Found: C, $56.70 ; \mathrm{H}, 4.06, \mathrm{~N}, 15.38$.

8-(4-Chlorophenyl)-1,2,3,4,7,8-hexahydro-9-nitropyrido[1,2-a] pyrimidin-6-one (5e). White solid; yield: $0.260 \mathrm{~g}(85 \%)$; mp 282$284{ }^{\circ} \mathrm{C} .{ }^{1} \mathrm{H}$ NMR $\left(300 \mathrm{MHz}, \mathrm{DMSO}-d_{6}\right): \delta 1.89-1.98\left(\mathrm{~m}, 2 \mathrm{H}, \mathrm{CH}_{2}\right)$, $2.65\left(\mathrm{~d},{ }^{2} J_{\mathrm{HH}}=15.0 \mathrm{~Hz}, 1 \mathrm{H}, \mathrm{CH}_{2}\right), 3.21\left(\mathrm{dd},{ }^{2} J_{\mathrm{HH}}=15.0 \mathrm{~Hz},{ }^{3} J_{\mathrm{HH}}\right.$ $\left.=7.5 \mathrm{~Hz}, 1 \mathrm{H}, \mathrm{CH}_{2}\right), 3.41-3.89\left(\mathrm{~m}, 4 \mathrm{H}, \mathrm{CH}_{2} \mathrm{NH}\right.$ and $\left.\mathrm{CH}_{2} \mathrm{~N}\right), 4.59$ $\left(\mathrm{d},{ }^{3} J_{\mathrm{HH}}=6.6 \mathrm{~Hz}, 1 \mathrm{H}, \mathrm{CH}\right), 7.15\left(\mathrm{~d},{ }^{3} \mathrm{~J}_{\mathrm{HH}}=8.4 \mathrm{~Hz}, 2 \mathrm{H}, \mathrm{Ar}\right), 7.31$ $\left(\mathrm{d},{ }^{3} J_{\mathrm{HH}}=7.2 \mathrm{~Hz}, 2 \mathrm{H}, \mathrm{Ar}\right), 11.55(\mathrm{~s}, 1 \mathrm{H}, \mathrm{NH}) .{ }^{13} \mathrm{C} \mathrm{NMR}(75.4$ MHz, DMSO- $\left.d_{6}\right): \delta 19.5,36.0,38.6,108.6,128.8,129.0,131.9$, 140.9, 152.9, 168.6. IR (KBr) $\left(\nu_{\max } / \mathrm{cm}^{-1}\right): 3089(\mathrm{NH}), 2927(\mathrm{CH})$, $1710(\mathrm{C}=\mathrm{O}), 1618(\mathrm{C}=\mathrm{C}), 1502$ and $1330\left(\mathrm{NO}_{2}\right), 1232(\mathrm{C}-\mathrm{N}) . \mathrm{MS}$ (EI, $70 \mathrm{eV}): m / z(\%)=307\left(\mathrm{M}^{+}, 23\right), 290$ (17), 261 (100), 196 (20), 178 (13), 151 (37), 115 (19), 84 (31), 56 (29). Anal. calc. for $\mathrm{C}_{14} \mathrm{H}_{14} \mathrm{ClN}_{3} \mathrm{O}_{3}$ (307.73): C, 54.64; H, 4.59; N, 13.65. Found: C, 54.26; H, 4.90; N, 13.49 .

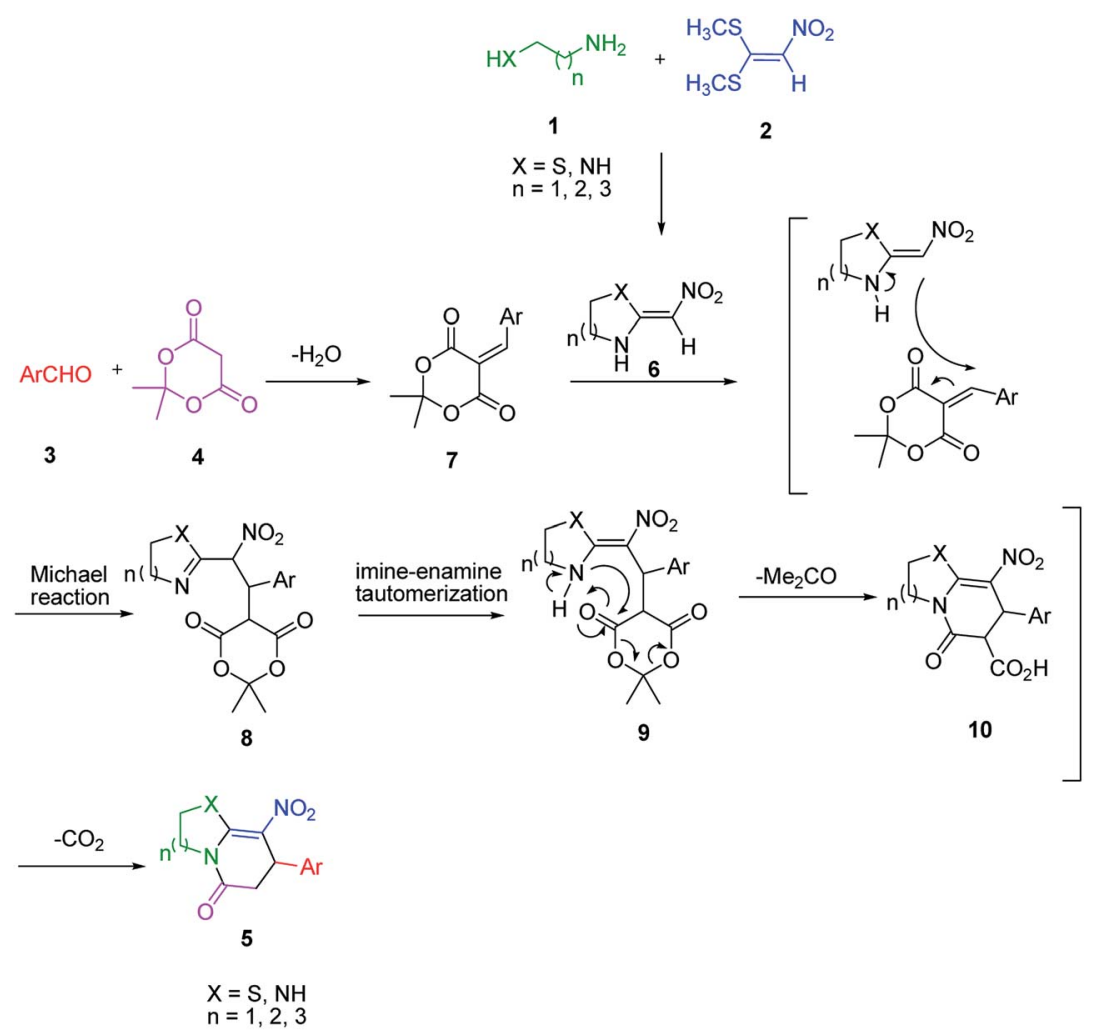

Scheme 3 Plausible mechanism for the formation of product 5. 

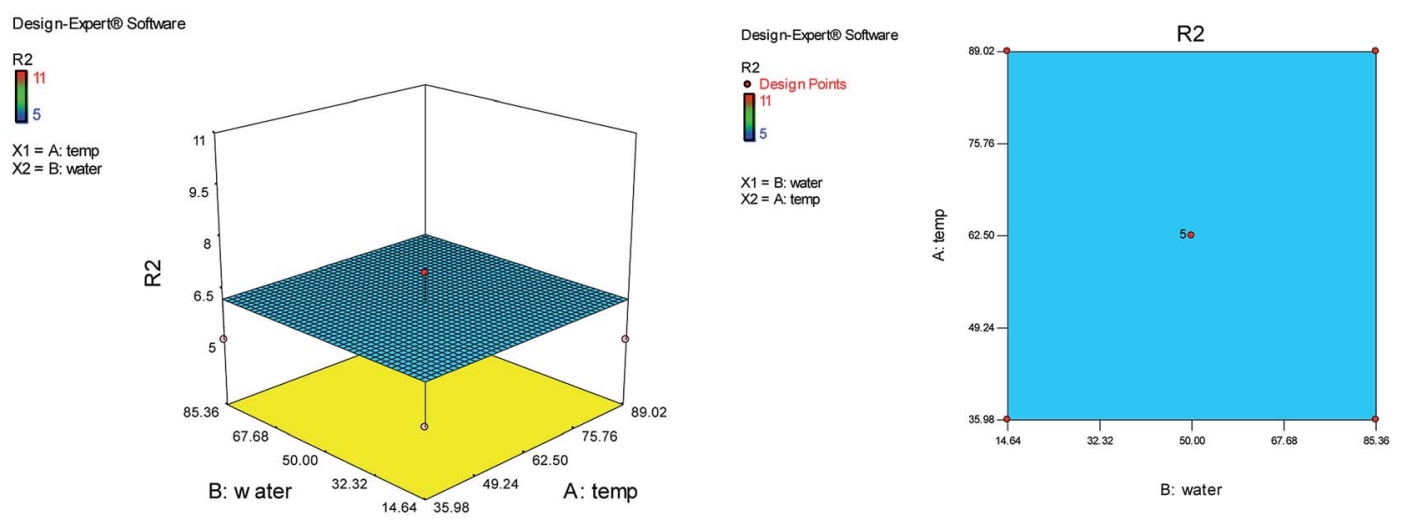

Fig. 3 The effects of temperature and the water content of aqueous ethanol on the total reaction time.

7-(4-Bromophenyl)-2,3,6,7-tetrahydro-8-nitrothiazolo[3,2-a]

pyridin-5-one (5f). Yellow solid; yield: $0.230 \mathrm{~g}$ (65\%); mp 204$206{ }^{\circ} \mathrm{C} .{ }^{1} \mathrm{H}$ NMR $\left(300 \mathrm{MHz}, \mathrm{DMSO}-d_{6}\right): \delta 2.60\left(\mathrm{~d},{ }^{2} J_{\mathrm{HH}}=16.5 \mathrm{~Hz}\right.$, $\left.1 \mathrm{H}, \mathrm{CH}_{2}\right), 3.17-3.39\left(\mathrm{~m}, 3 \mathrm{H}, \mathrm{CH}_{2}\right.$ and $\left.\mathrm{CH}_{2} \mathrm{~S}\right), 3.96-4.06(\mathrm{~m}, 1 \mathrm{H}$, $\left.\mathrm{CH}_{2} \mathrm{~N}\right), 4.32-4.39\left(\mathrm{~m}, 1 \mathrm{H}, \mathrm{CH}_{2} \mathrm{~N}\right), 4.64\left(\mathrm{~d},{ }^{3} \mathrm{~J}_{\mathrm{HH}}=8.4 \mathrm{~Hz}, 1 \mathrm{H}\right.$, $\mathrm{CH}), 7.14\left(\mathrm{~d},{ }^{3} J_{\mathrm{HH}}=8.4 \mathrm{~Hz}, 2 \mathrm{H}, \mathrm{Ar}\right), 7.36\left(\mathrm{~d},{ }^{3} J_{\mathrm{HH}}=8.4 \mathrm{~Hz}, 2 \mathrm{H}\right.$, Ar). ${ }^{13} \mathrm{C}$ NMR (75.4 MHz, DMSO- $\left.d_{6}\right): \delta 29.1,37.7,49.4,120.9$, 124.8, 129.0, 132.3, 140.3, 159.9, 166.6. IR (KBr) $\left(\nu_{\max } / \mathrm{cm}^{-1}\right)$ : $2918(\mathrm{CH}), 1699(\mathrm{C}=\mathrm{O}), 1573(\mathrm{C}=\mathrm{C}), 1420$ and $1350\left(\mathrm{NO}_{2}\right), 1223$ (C-N). MS (EI, $70 \mathrm{eV}): m / z(\%)=355\left(\mathrm{M}^{+}, 58\right), 339$ (100), 309 (40), 281 (17), 213 (16), 182 (22), 140 (18), 102 (21), 60 (15). Anal. calc. for $\mathrm{C}_{13} \mathrm{H}_{11} \mathrm{BrN}_{2} \mathrm{O}_{3} \mathrm{~S}$ (355.21): C, 43.96; H, 3.12; N, 7.89. Found: C, 43.56; H, 3.33; N, 8.26.

2,3,6,7-Tetrahydro-8-nitro-7-phenylthiazolo[3,2-a]pyridin-5-one (5g). Yellow solid; yield: $0.146 \mathrm{~g}(53 \%)$; mp $175-177^{\circ} \mathrm{C} .{ }^{1} \mathrm{H}$ NMR (300 MHz, DMSO- $\left.d_{6}\right): \delta 2.66\left(\mathrm{~d},{ }^{2} J_{\mathrm{HH}}=16.2 \mathrm{~Hz}, 1 \mathrm{H}, \mathrm{CH}_{2}\right), 3.25-$ $3.40\left(\mathrm{~m}, 3 \mathrm{H}, \mathrm{CH}_{2}\right.$ and $\left.\mathrm{CH}_{2} \mathrm{~S}\right), 3.95-4.05\left(\mathrm{~m}, 1 \mathrm{H}, \mathrm{CH}_{2} \mathrm{~N}\right), 4.33-4.41$ $\left(\mathrm{m}, 1 \mathrm{H}, \mathrm{CH}_{2} \mathrm{~N}\right), 4.63\left(\mathrm{~d},{ }^{3} \mathrm{~J}_{\mathrm{HH}}=7.5 \mathrm{~Hz}, 1 \mathrm{H}, \mathrm{CH}\right), 7.09-7.32(\mathrm{~m}$, $5 \mathrm{H}, \mathrm{Ar}) .{ }^{13} \mathrm{C}$ NMR $\left(75.4 \mathrm{MHz}, \mathrm{DMSO}-d_{6}\right): \delta 29.0,38.3,49.4,125.3$, 126.7, 127.8, 129.5, 140.9, 159.6, 166.8.

7-(4-Chlorophenyl)-2,3,6,7-tetrahydro-8-nitrothiazolo[3,2-a] pyridin-5-one (5h). Yellow solid; yield: $0.186 \mathrm{~g}(60 \%)$; mp 128$130{ }^{\circ} \mathrm{C} .{ }^{1} \mathrm{H}$ NMR $\left(300 \mathrm{MHz}, \mathrm{DMSO}-d_{6}\right): \delta 2.65\left(\mathrm{~d},{ }^{2} J_{\mathrm{HH}}=16.2 \mathrm{~Hz}\right.$, $\left.1 \mathrm{H}, \mathrm{CH}_{2}\right), 3.18-3.40\left(\mathrm{~m}, 3 \mathrm{H}, \mathrm{CH}_{2}\right.$ and $\left.\mathrm{CH}_{2} \mathrm{~S}\right), 3.96-4.06(\mathrm{~m}, 1 \mathrm{H}$, $\left.\mathrm{CH}_{2} \mathrm{~N}\right), 4.32-4.40\left(\mathrm{~m}, 1 \mathrm{H}, \mathrm{CH}_{2} \mathrm{~N}\right), 4.65\left(\mathrm{~d},{ }^{3} \mathrm{~J}_{\mathrm{HH}}=7.5 \mathrm{~Hz}, 1 \mathrm{H}\right.$, $\mathrm{CH}), 7.14\left(\mathrm{~d},{ }^{3} J_{\mathrm{HH}}=8.7 \mathrm{~Hz}, 2 \mathrm{H}, \mathrm{Ar}\right), 7.35\left(\mathrm{~d},{ }^{3} J_{\mathrm{HH}}=8.4 \mathrm{~Hz}, 2 \mathrm{H}\right.$, Ar). ${ }^{13} \mathrm{C}$ NMR (75.4 MHz, DMSO- $\left.d_{6}\right): \delta 29.1,37.7,49.5,124.9$, 128.7, 129.4, 132.4, 139.9, 159.9, 166.6. IR (KBr) $\left(\nu_{\max } / \mathrm{cm}^{-1}\right)$ : $2918(\mathrm{CH}), 1703(\mathrm{C}=\mathrm{O}), 1573(\mathrm{C}=\mathrm{C}), 1507$ and $1350\left(\mathrm{NO}_{2}\right), 1246$ $(\mathrm{C}-\mathrm{N})$.

2,3,6,7-Tetrahydro-8-nitro-7-(4-nitrophenyl)thiazolo[3,2-a] pyridin-5-one (5i). Yellow solid; yield: $0.182 \mathrm{~g}$ (57\%); mp 145$147{ }^{\circ} \mathrm{C} .{ }^{1} \mathrm{H}$ NMR $\left(300 \mathrm{MHz}, \mathrm{DMSO}-d_{6}\right): \delta 2.70\left(\mathrm{~d},{ }^{2} J_{\mathrm{HH}}=16.8 \mathrm{~Hz}\right.$, $\left.2 \mathrm{H}, \mathrm{CH}_{2}\right), 3.07-3.46\left(\mathrm{~m}, 3 \mathrm{H}, \mathrm{CH}_{2}\right.$ and $\left.\mathrm{CH}_{2} \mathrm{~S}\right), 3.99-4.09(\mathrm{~m}, 1 \mathrm{H}$, $\left.\mathrm{CH}_{2} \mathrm{~N}\right), 4.33-4.41\left(\mathrm{~m}, 1 \mathrm{H}, \mathrm{CH}_{2} \mathrm{~N}\right), 4.81\left(\mathrm{~d},{ }^{3} \mathrm{~J}_{\mathrm{HH}}=8.1 \mathrm{~Hz}, 1 \mathrm{H}\right.$, $\mathrm{CH}), 7.42\left(\mathrm{~d},{ }^{3} J_{\mathrm{HH}}=8.7 \mathrm{~Hz}, 2 \mathrm{H}, \mathrm{Ar}\right), 8.15\left(\mathrm{~d},{ }^{3} J_{\mathrm{HH}}=8.7 \mathrm{~Hz}, 2 \mathrm{H}\right.$, Ar). ${ }^{13} \mathrm{C}$ NMR (75.4 MHz, DMSO- $\left.d_{6}\right): \delta 29.1,38.2,49.5,124.2$, 124.6, 128.3, 147.2, 148.7, 160.4, 166.3. IR (KBr) $\left(\nu_{\max } / \mathrm{cm}^{-1}\right)$ : $2919(\mathrm{CH}), 1709(\mathrm{C}=\mathrm{O}), 1573(\mathrm{C}=\mathrm{C}), 1522$ and $1357\left(\mathrm{NO}_{2}\right), 1226$ (C-N). MS (EI, $70 \mathrm{eV}): m / z(\%)=321\left(\mathrm{M}^{+}, 36\right), 304$ (100), 274 (45), 246 (12), 199 (8), 140 (6), 60 (11). Anal. calc. for $\mathrm{C}_{13} \mathrm{H}_{11} \mathrm{~N}_{3} \mathrm{O}_{5} \mathrm{~S}$
(321.04): C, 48.59; H, 3.45; N, 13.08. Found: C, 48.79; H, 3.82; N, 13.50 .

2,3,6,7-Tetrahydro-8-nitro-7-phenylimidazo[1,2-a]pyridin5(1H)-one (5j). White solid; yield: $0.196 \mathrm{~g}$ (76\%); $\mathrm{mp} 266-268^{\circ} \mathrm{C}$. ${ }^{1} \mathrm{H}$ NMR (300 MHz, DMSO- $\left.d_{6}\right): \delta 2.55\left(\mathrm{~d},{ }^{2} J_{\mathrm{HH}}=16.2 \mathrm{~Hz}, 1 \mathrm{H}\right.$, $\left.\mathrm{CH}_{2}\right), 3.22\left(\mathrm{dd},{ }^{2} J_{\mathrm{HH}}=16.2 \mathrm{~Hz},{ }^{3} J_{\mathrm{HH}}=7.5 \mathrm{~Hz}, 1 \mathrm{H}, \mathrm{CH}_{2}\right), 3.73-$ $3.96\left(\mathrm{~m}, 4 \mathrm{H}, \mathrm{CH}_{2} \mathrm{NH}\right.$ and $\left.\mathrm{CH}_{2} \mathrm{~N}\right), 4.51\left(\mathrm{~d},{ }^{3} J_{\mathrm{HH}}=7.2 \mathrm{~Hz}, 1 \mathrm{H}, \mathrm{CH}\right)$, 7.02-7.29 (m, 5H, Ar), 9.73 (s, 1H, NH). ${ }^{13} \mathrm{C}$ NMR (75.4 MHz, DMSO- $\left.d_{6}\right): \delta 37.6,43.0,43.9,106.6,126.8,127.3,129.1,142.9$, 153.1, 167.8 .

2,3,6,7-Tetrahydro-7-(4-methoxyphenyl)-8-nitroimidazo[1,2-a] pyridin-5(1H)-one (5k). White solid; yield: $0.202 \mathrm{~g}(70 \%) ; \mathrm{mp}$ 307-309 ${ }^{\circ} \mathrm{C} .{ }^{1} \mathrm{H}$ NMR $\left(300 \mathrm{MHz}\right.$, DMSO- $\left.d_{6}\right): \delta 2.55\left(\mathrm{~d},{ }^{2} J_{\mathrm{HH}}=\right.$ $16.5 \mathrm{~Hz}, 1 \mathrm{H}, \mathrm{CH}_{2}$ ), $3.14\left(\mathrm{dd},{ }^{2} J_{\mathrm{HH}}=16.5 \mathrm{~Hz},{ }^{3} J_{\mathrm{HH}}=7.8 \mathrm{~Hz}, 1 \mathrm{H}\right.$, $\mathrm{CH}_{2}$ ), $3.68\left(\mathrm{~s}, 1 \mathrm{H}, \mathrm{OCH}_{3}\right), 3.72-3.97\left(\mathrm{~m}, 4 \mathrm{H}, \mathrm{CH}_{2} \mathrm{NH}\right.$ and $\left.\mathrm{CH}_{2} \mathrm{~N}\right)$, $4.32\left(\mathrm{~d},{ }^{3} \mathrm{~J}_{\mathrm{HH}}=7.5 \mathrm{~Hz}, 1 \mathrm{H}, \mathrm{CH}\right), 6.81\left(\mathrm{~d},{ }^{3} J_{\mathrm{HH}}=7.5 \mathrm{~Hz}, 2 \mathrm{H}, \mathrm{Ar}\right)$, $7.03\left(\mathrm{~d},{ }^{3} \mathrm{~J}_{\mathrm{HH}}=7.8 \mathrm{~Hz}, 2 \mathrm{H}, \mathrm{Ar}\right), 9.69(\mathrm{~s}, 1 \mathrm{H}, \mathrm{NH}) .{ }^{13} \mathrm{C} \mathrm{NMR}(75.4$ MHz, DMSO- $\left.d_{6}\right): \delta 36.7,43.0,43.9,55.4,107.0,114.4,127.8$, 134.7, 153.0, 158.6, 167.9.

2,3,6,7-Tetrahydro-7-(3-methoxyphenyl)-8-nitroimidazo[1,2-a] pyridin-5(1H)-one (5l). White solid; yield: $0.213 \mathrm{~g}$ (74\%); mp 213$215{ }^{\circ} \mathrm{C} .{ }^{1} \mathrm{H}$ NMR (300 MHz, DMSO- $\left.d_{6}\right): \delta 2.56\left(\mathrm{~d},{ }^{2} J_{\mathrm{HH}}=16.5 \mathrm{~Hz}\right.$, $\left.1 \mathrm{H}, \mathrm{CH}_{2}\right), 3.14\left(\mathrm{dd},{ }^{2} J_{\mathrm{HH}}=16.5 \mathrm{~Hz},{ }^{3} \mathrm{~J}_{\mathrm{HH}}=8.1 \mathrm{~Hz}, 1 \mathrm{H}, \mathrm{CH}_{2}\right), 3.69$ (s, $\left.1 \mathrm{H}, \mathrm{OCH}_{3}\right), 3.79-3.97\left(\mathrm{~m}, 4 \mathrm{H}, \mathrm{CH}_{2} \mathrm{NH}\right.$ and $\left.\mathrm{CH}_{2} \mathrm{~N}\right), 4.49(\mathrm{~d}$, $\left.{ }^{3} J_{\mathrm{HH}}=7.5 \mathrm{~Hz}, 1 \mathrm{H}, \mathrm{CH}\right), 6.66(\mathrm{~s}, 2 \mathrm{H}, \mathrm{Ar}), 6.77\left(\mathrm{~d},{ }^{3} J_{\mathrm{HH}}=7.2 \mathrm{~Hz}\right.$, $1 \mathrm{H}, \mathrm{Ar}), 7.18\left(\mathrm{t},{ }^{3} J_{\mathrm{HH}}=8.1 \mathrm{~Hz}, 1 \mathrm{H}, \mathrm{Ar}\right), 9.72(\mathrm{~s}, 1 \mathrm{H}, \mathrm{NH}) .{ }^{13} \mathrm{C} \mathrm{NMR}$ (75.4 MHz, DMSO- $d_{6}$ ): $\delta$ 37.5, 43.0, 43.9, 55.3, 106.5, 112.2, 113.1, 118.6, 130.2, 144.5, 153.1, 159.9, 167.8 .

7-(Furan-2-yl)-2,3,6,7-tetrahydro-8-nitroimidazo[1,2-a]pyridin5(1H)-one (5m). White solid; yield: $0.141 \mathrm{~g}$ (57\%); mp 241$243{ }^{\circ} \mathrm{C} .{ }^{1} \mathrm{H}$ NMR (300 MHz, DMSO- $\left.d_{6}\right): \delta 2.67\left(\mathrm{~d},{ }^{2} J_{\mathrm{HH}}=16.5 \mathrm{~Hz}\right.$, $\left.1 \mathrm{H}, \mathrm{CH}_{2}\right), 3.10\left(\mathrm{dd},{ }^{2} J_{\mathrm{HH}}=16.5 \mathrm{~Hz},{ }^{3} J_{\mathrm{HH}}=7.5 \mathrm{~Hz}, 1 \mathrm{H}, \mathrm{CH}_{2}\right)$, 3.70-3.97 (m, $4 \mathrm{H}, \mathrm{CH}_{2} \mathrm{NH}$ and $\left.\mathrm{CH}_{2} \mathrm{~N}\right), 4.61\left(\mathrm{~d},{ }^{3} J_{\mathrm{HH}}=6.9 \mathrm{~Hz}, 1 \mathrm{H}\right.$, $\mathrm{CH}), 6.04$ (s, 1H, Ar), 6.31 (s, 1H, Ar), 7.50 (s, 1H, Ar), 9.63 (s, 1H, $\mathrm{NH}) .{ }^{13} \mathrm{C}$ NMR $\left(75.4 \mathrm{MHz}\right.$, DMSO- $\left.d_{6}\right): \delta 32.0,36.6,43.0,43.8$, 104.9, 105.7, 110.8, 142.6, 152.7, 154.7, 167.7.

2,3,6,7-Tetrahydro-7-(3-methoxyphenyl)-8-nitrothiazolo[3,2-a] pyridin-5-one (5n). Yellow solid; yield: $0.153 \mathrm{~g}$ (50\%); mp 130$132{ }^{\circ} \mathrm{C} .{ }^{1} \mathrm{H}$ NMR $\left(300 \mathrm{MHz}, \mathrm{DMSO}-d_{6}\right): \delta 2.70\left(\mathrm{~d},{ }^{2} J_{\mathrm{HH}}=16.5 \mathrm{~Hz}\right.$, $\left.1 \mathrm{H}, \mathrm{CH}_{2}\right), 3.27-3.40\left(\mathrm{~m}, 3 \mathrm{H}, \mathrm{CH}_{2}\right.$ and $\left.\mathrm{CH}_{2} \mathrm{~S}\right), 3.70\left(\mathrm{~s}, 1 \mathrm{H}, \mathrm{OCH}_{3}\right)$, 3.93-4.03 (m, $\left.1 \mathrm{H}, \mathrm{CH}_{2} \mathrm{~N}\right), 4.33-4.41\left(\mathrm{~m}, 1 \mathrm{H}, \mathrm{CH}_{2} \mathrm{~N}\right), 4.61\left(\mathrm{~d},{ }^{3} J_{\mathrm{HH}}\right.$ 
$=7.2 \mathrm{~Hz}, 1 \mathrm{H}, \mathrm{CH}), 6.61-6.83(\mathrm{~m}, 3 \mathrm{H}, \mathrm{Ar}), 7.21\left(\mathrm{t},{ }^{3} \mathrm{~J}_{\mathrm{HH}}=7.8 \mathrm{~Hz}\right.$, $1 \mathrm{H}, \mathrm{Ar}) .{ }^{13} \mathrm{C}$ NMR (75.4 MHz, DMSO- $\left.d_{6}\right): \delta 29.0,38.2,49.4,55.4$, 112.8, 112.9, 118.3, 125.1, 130.6, 142.5, 159.6, 160.1, 166.8.

2,3,6,7-Tetrahydro-8-nitro-7-propylimidazo[1,2-a]pyridin-5(1H)one (5o). White solid; mp 174-176 ${ }^{\circ} \mathrm{C} .{ }^{1} \mathrm{H}$ NMR $(300 \mathrm{MHz}$, DMSO- $\left.d_{6}\right): \delta 0.83\left(\mathrm{t}, 3 \mathrm{H}, \mathrm{CH}_{3}\right), 1.15-1.45\left(\mathrm{~m}, 4 \mathrm{H}, 2 \mathrm{CH}_{2}\right), 2.42(\mathrm{~d}$, $\left.{ }^{2} J_{\mathrm{HH}}=16.8 \mathrm{~Hz}, 1 \mathrm{H}, \mathrm{CH}_{2}\right), 2.77\left(\mathrm{dd},{ }^{2} J_{\mathrm{HH}}=16.8 \mathrm{~Hz},{ }^{3} J_{\mathrm{HH}}=\right.$ $\left.7.2 \mathrm{~Hz}, 1 \mathrm{H}, \mathrm{CH}_{2}\right), 3.62-3.93\left(\mathrm{~m}, 5 \mathrm{H}, \mathrm{CH}\right.$ and $\mathrm{CH}_{2} \mathrm{NH}$ and $\left.\mathrm{CH}_{2} \mathrm{~N}\right)$, $9.54(\mathrm{~s}, 1 \mathrm{H}, \mathrm{NH})$.

\section{Conclusion}

We have reported a simple and clean synthesis of imidazopyridinone, pyridopyrimidinone, and thiazolopyridinone derivatives. We were able to optimize the reaction conditions using RSM in conjunction with a central composite design. Further experimentation revealed the optimal values of the water content of aqueous ethanol (33\%), and reaction temperature (72 ${ }^{\circ} \mathrm{C}$ ). Also good yield of product in mild conditions show that the experimental results are in good agreement with the predicted values, and the model successfully can be used to predict the synthesis of such heterocyclic compounds.

\section{Conflicts of interest}

The authors declare no competing financial interest.

\section{Acknowledgements}

We gratefully acknowledge the financial support of this research from Imam Khomeini International University. We thank the Iran National Science Foundation (No. 97021367) for financial support.

\section{Notes and references}

1 D. Bas and I. H. Boyaci, J. Food Eng., 2007, 78, 836-845.

2 H. Tang, Q. Xiao, H. Xu and Y. Zhang, Org. Process Res. Dev., 2013, 17, 632-640.

3 J. Palaniraja, S. M. Roopan and G. M. Rayalu, $R S C A d v ., 2016$, 6, 24610-24616.

4 B. Maherani, M. Barzegar and M. A. Sahari, J. Food Sci. Technol., 2007, 44, 250-254.

5 V. K. Garlapati and L. Roy, J. Young Pharm., 2017, 9, 417-421. 6 A. Ryad, K. Lakhdar, K. S. Majda, A. Samia, A. Mark, A. D. Corinne and G. Eric, Int. J. Mol. Sci., 2010, 11, 47264740.

7 I. R. Parrey and A. A. Hashmi, Catal. Sustainable Energy, 2016, 3, 1-6.

8 L. Yurttas, Y. Ozkay, H. K. Gencer and U. Acar, J. Chem., 2015, 2015, 1-7.

9 Y. Cui and P. E. Floreancig, Org. Lett., 2012, 14, 1720-1723. 10 A. Lozynskyi, B. Zimenkovsky, L. Radko, S. Stypula-Trebas, O. Roman, A. K. Gzella and R. Lesyk, Chem. Pap., 2018, 72, 669-681.
11 G. M. Ziarani, N. H. Nasab, M. Rahimifard and A. A. Soorki, J. Saudi Chem. Soc., 2015, 19, 676-681.

12 S. M. Roopan, S. M. Patil and J. Palaniraja, Res. Chem. Intermed., 2016, 42, 2749-2790.

13 L. R. Wen, Z. R. Li, M. Li and H. Cao, Green Chem., 2012, 14, 707-716.

14 S. H. Li, Y. H. Shen, N. Gao and J. T. Li, Eur. J. Chem., 2010, 7, 779-784.

15 I. Yavari, M. Nematpour and E. Sodagar, Monatsh. Chem., 2015, 146, 2135-2138.

16 M. Mamaghani and R. Hossein Nia, J. Heterocycl. Chem., 2017, 54, 1700-1722.

17 S. Abdolmohammadi and M. Afsharpour, Chin. Chem. Lett., 2012, 23, 257-260.

18 K. C. Majumdar, S. Ponra and D. Ghosh, Synthesis, 2011, 1132-1136.

19 A. M. Rad and M. Mokhtary, Int. Nano Lett., 2015, 5, 109-123. 20 A. H. Shamroukh, A. E. Rashad and F. M. E. Abdelmegeid, J. Chem. Pharm. Res., 2016, 8, 734-772.

21 M. Boominathan, M. Nagaraj, C. Maheshwaran, S. Muthusubramanian and N. Bhuvanesh, J. Heterocycl. Chem., 2014, 51, 244-248.

22 G. A. El-Hiti, Monatsh. Chem., 2003, 134, 837-841.

23 M. E. Azab, G. A. M. El-Hag Ali and A. H. F. Abd El-Wahab, Acta Pharm., 2003, 53, 213-221.

24 H. L. Chen and H. Y. Guo, J. Chem. Res., 2012, 36, 162-165. 25 F. Shi, C. Li, M. Xia, K. Miao, Y. Zhao, S. Tu, W. Zheng, G. Zhang and N. Ma, Bioorg. Med. Chem. Lett., 2009, 19, 5565-5568.

26 R. A. Mekheimer, N. M. Hilmy, A. A. Hameed, S. Dacrory and K. U. Sadek, Synth. Commun., 2011, 41, 2511-2516.

27 K. M. Wang, S. J. Yan and J. Lin, Eur. J. Org. Chem., 2014, 1129-1145.

28 P. H. Yang, Res. Chem. Intermed., 2016, 42, 5617-5637.

29 L. Pan, X. Bi and Q. Liu, Chem. Soc. Rev., 2013, 42, 1251-1286.

30 I. Yavari, N. Zahedi, L. Baoosi and S. Skoulika, Mol. Diversity, 2018, 22, 11-19.

31 L. Zhang, J. Dong, X. Xu and Q. Liu, Chem. Rev., 2016, 116, 287.

32 D. P. Sangi, J. L. Monteiro, K. L. Vanzolini, Q. B. Cass, M. W. Paixao and A. G. Correa, J. Braz. Chem. Soc., 2014, 25, 887-889.

33 D. Elumalai, R. Gnanasekaran, S. Leelakrishnan, G. Nachimuthu, T. Kannan, T. P. Paramasivam and K. Jayabal, ChemistrySelect, 2018, 3, 2070-2079.

34 S. K. Movahed, M. Dabiri and A. Bazgir, Helv. Chim. Acta, 2013, 96, 525-532.

35 M. Yildirim, D. Celikel, N. Evis, D. W. Knight and B. M. Kariuki, Tetrahedron, 2014, 70, 5674-5681.

36 M. Bayat and F. S. Hosseini, Tetrahedron Lett., 2017, 58, 1616-1621.

37 M. Bayat and F. S. Hosseini, J. Sulfur Chem., 2018, 39, 279293.

38 M. Bayat and M. Rezaei, J. Iran. Chem. Soc., 2018, 15, 769777. 